\title{
Los sistemas de información geográfica en la geografía médica*
}

\author{
Carlos Garrocho \\ El Colegio Mexiquense
}

...the point of all science, indeed all learning and reflection, is to change and develop our understanding and reduce illusion.

Andrew Sayer

\section{Resumen}

En este documento se exploran algunas posibilidades de los sistemas de información geográfica (SIG) para apoyar la investigación en geografía médica. Las dos primeras secciones son de corte conceptual: en la primera se define la disciplina, otorgando especial atención a sus diferentes objetos de estudio; en la segunda se define la herramienta, con la finalidad de identificar sus posibilidades técnicas y analíticas. Una vez que se establece el significado de los dos principales componentes de este trabajo-geografía médica y SIG-, se procede, en la tercera sección, a examinar cómo la herramienta puede apoyar la exploración de las preguntas centrales de la disciplina. Finalmente, en la última sección se plantean las principales conclusiones del documento.

* Una primera versión se presentó en el vn Congreso Nacional de Investigación en Salud Pública de México, 3 a 5 de marzo de 1997, Instituto Nacional de Salud Pública, Cuernavaca, Morelos. Agradezco el valioso apoyo de la Facultad de Arquitectura y Diseño de la Universidad Autónoma del Estado de México para la realización de esta ponencia. 
Introducción

En años recientes la geografía médica tradicional -orientada preferentemente a estudiar la ecología de las enfermedades- ha sido complementada por estudios de corte espacial relacionados con aspectos más generales del proceso salud-enfermedad y la relación oferta-demanda de los servicios de salud. Estos nuevos intereses conforman lo que Phillips (1981) llama geografía sociomédica, una de las ramas más dinámicas de la geografía contemporánea.

También en años recientes - pero en el campo de la informática- han surgido notables recursos tecnológicos y poderosas herramientas de análisis espacial, inimaginables pocos años atrás. En México, apenas en la segunda mitad de la década de los ochenta comenzó a popularizarse la utilización de computadoras personales (PC) en los centros de investigación en ciencias sociales. Los paquetes más utilizados eran las hojas de cálculo (Lotus 1-2-3, por ejemplo), los procesadores de palabras (como el viejo MemoMaker), y en menor grado algunos de análisis estadístico (como el Statistical Package for Social Sciences, mejor conocido como SPSS).

Las entonces nuevas y sorprendentes posibilidades gráficas, de cálculo y de escritura, permitieron el trabajo mucho más eficiente $-\mathrm{y}$ quizá más efectivo, aunque esto está abierto a debate- de los geógrafos y de todos los científicos sociales en general. Pero - por si no fueran suficientes esos notables adelantos tecnológicos- la informática les tenía deparada una gran sorpresa a los geógrafos: los SIG.

Los SIG son relativamente nuevos, y para todo fin práctico, los geógrafos mexicanos comenzamos a utilizar los SIG hace apenas unos cuantos años. El resultado es a menudo un coctel peligroso, cuyos principales ingredientes son: inexperiencia, ilusión y confusión. Inexperiencia, porque apenas empezamos a contar con personal verdaderamente capacitado en el manejo eficiente de los SIG - aunque ya se enseña a manejarlos en algunas licenciaturas-;' ilusión, porque las posibilidades visuales de los SIG con frecuencia generan expectativas desmedidas acerca de los beneficios que se derivarán de su utilización -aunque sólo en raras ocasiones se pueden satisfacer cabalmente-; y confusión, porque no es raro encontrar estudiantes $\mathrm{e}$ incluso investigadores profesionales que definen sus intereses académicos en función de las posibilidades de los SIG que saben

'Por ejemplo, en la Facultad de Geografía y en la de Planeación Urbana y Regional, ambas de la Universidad Autónoma del Estado de México. manejar o que tienen a su alcance -confundiendo el medio, es decir el SIG, con el fin, o sea, abordar problemas de investigación socialmente relevantes.

Resumiendo, este es el contexto: una disciplina dinámica con una gran tradición académica (la geografía médica) se encuentra con una de las herramientas de análisis espacial más poderosas de todos los tiempos (los SIG). Esta situación, aparentemente nueva, tiene un notable parecido con lo que le ocurrió a la geografía en los años cincuenta y sesenta. En esas décadas, los geógrafos descubrieron la luz del análisis cuantitativo formal, y las posibilidades analíticas - reales y ficticias- que de pronto tuvieron ante sí los sedujeron, pero además los deslumbraron (Clarke y Wilson, 1989).

En esos años, los geógrafos produjeron un cúmulo de investigaciones cuantitativas, aunque no siempre generaron buena investigación. Al margen de sus innegables logros, aún se recuerdan algunos de los graves errores epistemológicos de esa revolución cuantitativa: negación de lo cualitativo, atención exclusiva a lo cuantificable, confusión de los medios con los fines. La lección a rescatar es que cuando se abusó del recurso el resultado fue negativo: trabajos complejos y rigurosos en términos cuantitativos, pero socialmente irrelevantes.

Ahora -en la segunda mitad de los años noventa- me parece que los geógrafos estamos ante una nueva realidad: la revolución informática. La computadora -y particularmente los SIG- están deslumbrando y seduciendo a los geógrafos, tal como les ocurrió con el análisis cuantitativo hace casi cuarenta años.

Para no cometer los mismos errores, los geógrafos debemos ser más reflexivos sobre las posibilidades reales que tienen las nuevas herramientas automatizadas para apoyar nuestras tareas de investigación. Deberíamos recordar quelainvestigación comienza con preguntas socialmente relevantes, no con herramientas casualmente disponibles.

\section{¿Qué son los sistemas de información geográfica?}

Los SIG son una clase especial de sistema compartido de información (Álvarez, 1997). Aunque resulta difícil dar una definición universal de los SIG, para los propósitos de este trabajo se podría decir que son manejadores de base de datos automatizados capaces de almacenar, recuperar, analizar y desplegar información, actividades o eventos 
georreferenciados, en forma tabular, gráfica y cartográfica (Huxhold 1991).

En la literatura especializada, los SIG son entendidos de tres maneras básicas: $i$ ) como generadores de mapas, ii) como manejadore cartográficos de bases de datos, y iii) como herramientas de análisis espacial (Álvarez, 1997). Como generador de mapas un SIG es simplemente una medio de captura, despliegue y procesamiento cartográfico. Cada conjunto de datos es representado como un mapa en sí mismo (también llamado capa o tema), y los mapas pueden ser combinados o superpuestos para generar nueva información cartográfica. Es decir, un nuevo mapa.

La visión de los SIG como base de datos pone énfasis en el diseño e instrumentación de las relaciones entre archivos de datos (Frank, 1988). Por lo tanto, el administrador de bases de datos se convierte en parte integral del SIG. Las aplicaciones de los SIG como bases de datos normalmente implican búsquedas complejas que luego se expresan cartográficamente. Es posible incorporar otras operaciones analíticas más complejas, pero a menudo se requiere resolver algunos problemas de programación (Garrocho, 1997a).

La tercera visión de los SIG subraya la importancia del análisis espacial: por ejemplo, estadística espacial, diversas técnicas de análisis geográfico y modelado de procesos socioespaciales. ${ }^{2}$ Desde este punto de vista, los SIG son considerados más como laboratorios de ambiente controlado, que como simples herramientas analíticas (Maguire, Goodchild y Rhind, 1991).

La conclusión es evidente, los SIG pueden ser entendidos de diferentes maneras dependiendo de la perspectiva del usuario y del uso que se les pretenda dar.

\section{Elementos básicos de los SIG}

Un SIG está compuesto de cuatro elementos básicos interrelacionados: el equipo de cómputo (hardware), los programas (software), los datos, y el usuario capacitado (liveware). Los elementos de hardware pueden ser de muchos tipos, desde las sencillas computadoras personales, hasta las estaciones de trabajo de alto desempeño, minicomputadoras y mainframes. En los años noventa prevalece la tendencia a utilizar estaciones de trabajo bajo el sistema operativo UNIX, aunque existen en el mercado productos que operan en computadora personal bajo ambiente Windows y que compiten con las más costosas estaciones de trabajo UNIX. Adicionalmente, los dispositivos de entrada, almacén, y salidas, son elementos esenciales de periféricos para captura de datos (por ejemplo scanners, tabletas digitalizadoras, unidades de respaldo) y salidas de información (por ejemplo graficadores, impresoras láser o de inyección de tinta, etcétera).

Los programas de cómputo (software) son quizás la parte más visible de lo que es un SIG. Incluso, con frecuencia los especialistas en computación, y en general los que no lo son, tienden a pensar en los SIG como paquetes de software. Sin entrar en demasiados detalles, el software de un SIG debe ser capaz -entre otras cosas- de crear, almacenar, editar, manipular y desplegar información cartográfica; transformar coordenadas, cambiar escalas, calcular áreas de regiones y distancias entre puntos; asignar y almacenar atributos de diversos tipos (números, textos, gráficas) de cualquier punto, línea o región de un mapa; ordenar, buscar, relacionar o combinar datos georreferenciados; relacionar espacialmente los datos cartográficos, es decir, reconocer objetos que estén a la derecha, izquierda o junto a cierto objeto, o reconocer la intersección de dos líneas o polígonos; analizar espacialmente los datos, esto es, determinar patrones de datos asociados con su localización y manipularlos para derivar nueva información a partir de los datos existentes.

Los datos, por su parte, resultan muy caros de recolectar, almacenar y manipular, debido al gran volumen de información que se requiere para resolver problemas concretos. Se estima que el costo de los datos excede al costo del hardware y software en una proporción de 2 a 1 , y se ha calculado que los datos representan $70 \%$ del costo total de la instrumentación de un SIG (Álvarez, 1997). Sin embargo, la situación está cambiando. Por un lado, existe una demanda intensa que clama por información; y por el otro, diversas firmas compitiendo por el mercado, ofreciendo grandes volúmenes de datos georreferenciados en medios electrónicos a costos cada vez más accesibles. Las bases de datos del Instituto Nacional de Estadística, Geografía e Informática (INEGI) son una clara muestra de esta tendencia.

El cuarto y último elemento fundamental de los SIG es el personal responsable de diseñarlo, implantarlo y usarlo. Sin un personal adecuadamente capacitado, difícilmente se pueden alcanzar la metas planteadas en cualquier proyecto de desarrollo de SIG. Aunque parezca obvio, la falta de entrenamiento y experiencia es un aspecto que debe ser privilegiado si se quiere tener éxito con los SIG. 
Basta una mala selección de hardware y software, una incorrecta digitalización y georreferencia de los datos, o una mala selección de fuentes de información - todas estas decisiones las toma el responsable del SIG-, para llevar al fracaso la instrumentación de esta herramienta.

\section{Aplicaciones de los SIG}

Los SIG pueden ayudar a contestar diversos tipos de preguntas. En la literatura especializada se pueden identificar cuando menos las siguientes: de localización (¿qué hay en cierto lugar?); condición (¿dónde hay, está u ocurrió alguna cosa o evento?); tendencia (¿qué ha cambiado en cierto sitio?); ruta (¿cuál es el trayecto más conveniente para llegar a cierto lugar, saliendo de determinado punto?); patrones (¿qué es común a determinados sitios?); y modelado (¿que pasaría en determinados lugares si cambian determinadas variables?).

Las preguntas de localización involucran búsquedas en bases de datos para estimar el tipo de fenómeno que puede ocurrir en un lugar determinado. Las de condición pretenden encontrar los sitios que tienen ciertas características comunes. Las de tendencia permiten monitorear cambios en determinadas variables en un cierto tiempo. Las preguntas de ruta requieren la implantación de algoritmos de optimización de trayectos, y las de patrones implican cálculos estadísticos complejos para describir, comparar y explicar el comportamiento espacial de fenómenos concretos. Para explorar el último tipo de pregunta - de modelado- se utilizan modelos matemáticos que permitan generar escenarios posibles y probables ("parecidos a la realidad").

Las posibilidades analíticas de los SIG son múltiples. Se utilizan de manera intensiva en el sector privado para planear estrategias de mercado, y en el sector público y académico contribuyen de manera valiosa para apoyar el entendimiento y solución de problemas sociales, económicos, ambientales $\mathrm{y}$, en general, de desarrollo urbano y regional. El interés en los SIG está creciendo rápidamente y es razonable esperar una evolución aún más acelerada de esta tecnología en los años por venir.

\section{¿Qué es la geografía médica?}

Si se acepta que el medio ambiente - tanto físico como social- afecta la salud de la población, entonces se justifica estudiar la distribución espacial de los problemas de salud y tratar de explicarlos, aunque sea de manera parcial, a partir de las condiciones particulares del entorno. Esto es lo que intenta hacer la geografía médica tradicional: describir detalladamente patrones espaciales de morbilidad y mortalidad.

En años recientes, sin embargo, la geografía médica ha ampliado su interés al estudio de los sistemas de salud, particularmente en lo que se refiere a su distribución socioespacial, accesibilidad, utilización y calidad (Joseph y Phillips, 1985). La corriente tradicional de la geografía médica ha sido llamada "ecológica" (Learmonth, 1978), y la contemporánea, de "atención a la salud" (o "geography of health care”, Phillips, 1981). Aunque esta segunda corriente es cada vez más importante en la literatura internacional, es necesario subrayar que los dos enfoques se complementan, no se sustituyen; conforman de manera integral la misma disciplina, y son igualmente relevantes (Meade, Florin y Gesler, 1988).

\section{Principales especialidades en geografía médica}

Es posible distinguir diversas especialidades en geografía médica, y en esta sección se examinan brevemente las principales, con el fin de definir una imagen general de la disciplina.

Cartografía médica. La representación en mapas de patrones espaciales de la salud y la enfermedad es una de las perspectivas académicas de mayor tradición en la geografía médica. Incluso, generó las primeras contribuciones importantes de los geógrafos al estudio del proceso salud-enfermedad. Algunos de los trabajos más conocidos de los geógrafos médicos son precisamente de corte cartográfico, por ejemplo, los numerosos Atlas de Salud publicados desde hace mucho tiempo en diversas partes del mundo.

Ecología médica. La perspectiva ecológica se desarrolló aceleradamente después de la Segunda Guerra Mundial, sobre todo, a partir de las contribuciones de May (1958), quien brindó especial atención a la relación espacial entre los "factores patológicos" y los "factores geográficos" para explicar la salud diferencial de grupos humanos.

May identificó ocho factores fundamentales para la salud; cinco patológicos y tres geográficos. Los factores patológicos eran los siguientes: agentes causativos (microorganismos); transmisores de agentes causativos (por ejemplo, moscas, pulgas, mosquitos); receptores intermedios (organismos esenciales para el ciclo de vida de los agentes causativos); reservas (receptores animales que cargan la 
infección y que eventualmente la transmiten al hombre); $\mathrm{y}$, finalmente, el ser humano (el último elemento de la compleja cadena patológica). Por su parte, May sintetizó los factores geográficos como: medio ambiente físico inorgánico (clima, latitud, temperatura, humedad, y otros); medio ambiente sociocultural (distribución del ingreso, perfil demográfico, niveles de vida, calidad de la vivienda, etcétera); y medio ambiente biológico orgánico (flora y fauna, principalmente).

Otros autores (Pyle, 1976 y 1977 y McGlashan, 1966) incorporaron avanzados análisis estadísticos a la estructura ecológica de May, generando investigaciones más rigurosas y complejas, que algunos llaman "estudios asociativos" (Meade, Florin y Gesler, 1988). Cuando además se incorpora información social georreferenciada de manera intensiva - como lo hace Giggs (1983)-, entonces reciben el nombre de "estudios de áreas sociales".

Sin embargo, los tres tipos de estudios (ecológicos, asociativos, y de áreas sociales) tienen en común su poco poder de explicación. Incapaces de establecer la relación causa-efecto, se limitan a presentar asociaciones estadísticas - que a veces han resultado espurias- entre factores de riesgo y patrones de salud. No obstante, no hay que minimizar el valor descriptivo del enfoque ecológico como insumo de las políticas de salud pública. En este sentido merece un espacio aparte el enfoque llamado "epidemiología del paisaje".

Epidemiología del paisaje. Este enfoque fue desarrollado por el geógrafo ruso Eugene N. Pavlovski (1966), quien propuso que los factores de riesgo para la salud humana no se distribuían homogéneamente en el territorio, sino que se concentraban en áreas específicas que favorecían el ciclo de vida de algunas enfermedades. Pavlovski razonó que si fuera posible identificar estas áreas - que él llamó "zonas silenciosas"- entonces se podrían tomar medidas preventivas para evitar el surgimiento y la eventual transmisión de la enfermedad.

Pavlovski propuso que para identificar las zonas de riesgo de enfermedades específicas, se debería conocer primero el ciclo patológico de la enfermedad de referencia. Es decir, las condiciones (climáticas, topográficas, de flora y fauna, la composición química de suelos, y otros) que favorecen el desarrollo y la transmisión de los agentes causales. Los razonamientos de Pavlovski fueron traducidos en políticas de salud pública y resultaron fundamentales en la planeación y fundación de nuevos asentamientos en Siberia (Meade, Florin y Gesler, 1988). La identificación de zonas de riesgo debe ser considerada parte importante en términos de salud pública, porque permite concentrar los recursos y esfuerzos destinados a la prevención de enfermedades.

Estudios de difusión. A diferencia de los estudios ecológicos tradicionales, los de difusión no pretenden establecer patrones espaciales de salud y enfermedad en un tiempo dado, sino estudiar o modelar la difusión espacial y temporal de las enfermedades o de fenómenos médicos. ${ }^{3}$ Los geógrafos han utilizado diversos métodos analíticos en sus estudios de difusión: inspección visual de mapas, modelos teóricos y matemáticos, y técnicas de estadística espacial (Pyle, 1986). Es importante conocer cómo se difunden en el espacio las enfermedades para tomar medidas anticipadas a su aparición en lugares específicos. Los estudios de difusión a menudo son predictivos y por lo tanto muy útiles en términos de planeación.

Estudios de distribución de servicios. Los estudios de distribución de servicios de salud tienen profundas raíces morales, derivadas de las teorías de "justicia distributiva" (Garrocho, 1993c).El propósito de estos estudios es identificar desigualdades injustificables en la distribución socioespacial de los servicios de salud, y sugerir medidas correctivas. Las herramientas más importantes para realizar este tipo de análisis son los mapas que presentan indicadores de distribución de los servicios, así como las técnicas estadísticas de desigualdad socioespacial. ${ }^{4}$

Estudios de simulación y modelado. Aunque los estudios de difusión también podrían clasificarse en esta categoría, los de modelado y simulación se refieren más a la localización y utilización de los servicios de salud. Esta es una de las áreas de más reciente desarrollo en geografía médica, y una de las de mayor potencial para generar resultados traducibles en términos de políticas de salud pública (Garrocho, 1990a).

Los estudios de simulación y modelado son predominantemente cuantitativos, aunque los trabajos más recientes tratan de integrar en una misma estructura variables cuantitativas y cualitativas (Garrocho, 1995a). Dos tipos de modelos son los más utilizados por los geógrafos médicos cuando realizan estudios de

${ }^{3}$ Por ejemplo, la aplicación de ciertas prácticas médicas o innovaciones en medicina. Para utilizar un símil un tanto simple, mientras en los estudios ecológicos se pretende tomar una fotografia de la realidad en materia de salud y enfermedad, en los estudios de difusión se incierta enfermedad o ciertas prácticas médicas.

${ }^{4}$ Por ejemplo, camas o médicos por mil o diez mil habitantes, curvas de Lorenz, índices de Gini, y otros. 
simulación: los de localización-asignación (location-allocationmodels), para definir patrones de localización de los servicios, en función de cierto o ciertos criterios sociales (Garrocho, 1990b; 1992), y los de interacción espacial, para simular flujos de pacientes a unidades de salud - o como se registra en la geografía de las actividades comerciales-, esto es, la conducta espacial de los usuarios. Cabe decir que existen en nuestro país intentos de combinar modelos de ambos tipos en un solo esquema analítico (Garrocho, 1995a).

Estudios de utilización. Éstos buscan explicar por qué la población utiliza o no ciertos servicios médicos (Joseph y Phillips, 1985). Tales estudios son deliberadamente eclécticos e integran variables espaciales y no espaciales, cuantitativas y cualitativas, y diversos modelos discursivos y matemáticos (Garrocho, 1995a). Los estudios de utilización son complejos debido a las numerosas variables que se tienen que considerar, pero han demostrado ser muy útiles en términos de planeación de los servicios de salud (Garrocho, 1993a).

Aunque esta revisión de las principales especialidades de la geografía médica permite perfilar la disciplina, es necesario identificar sus preguntas fundamentales para definirla con mayor claridad, y evaluar el apoyo que pueden brindar los SIG para intentar responderlas,

\section{Las preguntas fundamentales de la geografía médica y el apoyo potencial de los SIG}

Tomando como punto de partida las investigaciones de autores como Amedeo y Golledge (1975), Phillips (1981), Joseph y Phillips (1985), y Meade, Florin y Gesler (1988), es posible identificar cuando menos cinco preguntas fundamentales en geografía médica. Al relacionar estas preguntas con la definición conceptual de los SIG - planteada en la primera sección de este documento- se puede responder la pregunta que le da sentido a este texto: ¿cuál es la utilidad potencial de los sistemas de información geográfica en la geografía médica?

\section{Preguntas de investigación de la geografía médica y apoyo potencial de los} SIG

i. ¿्Por qué los servicios de salud y los niveles de salud y enfermedad de la población se distribuyen espacialmente de determinada manera?

Basta revisar las estadísticas para notar las enormes diferencias internacionales, regionales eintraurbanas en materia de indicadores de salud (tasas de mortalidad, esperanza de vida, eventos de enfermedad por año, cobertura de vacunación contra enfermedades específicas) y de disponibilidad de servicios de salud (médicos, camas de hospital, incubadoras, medicinas).

El primer paso para explicar la distribución espacial de lasalud y los recursos para ésta, es definir con cuidado su localización. Es decir, sintetizarlos cartográficamente. Los mapas son una de las principales herramientas para comenzar los análisis de distribución espacial de variables relacionadas con la salud humana; tanto, que - como ya se dijo- algunas de las obras más conocidas de la geografía médica son los llamados Atlas de salud.

En este sentido, los SIG tienen un potencial enorme porque permiten generar numerosos mapas de gran calidad en muy poco tiempo. Aún más, facilitan actualizar los datos, lo que redunda en ahorros de tiempo, energía y dinero en el mantenimiento de la base de información cartográfica.

Los SIG pueden generar diversos tipos de mapas. Los más comunes son los temáticos, en los que se aplican texturas o colores sobre áreas específicas. Es importante que un mapa temátrco no tenga más de cinco tipos de texturas o colores, para facilitar su lectura y referencia. Un simple vistazo a un mapa temático permite captar más facilmente la información que si se observa un cuadro, y facilita identificar claramente las diferencias regionales en materia de indicadores de salud o de disponibilidad de servicios en la materia.

Evidentemente, los datos deberán estandarizarse respecto a la población de cada área que integra el mapa, y es recomendable usar indicadores que ilustren cuando menos tres aspectos: la magnitud de las variables (por ejemplo, doctores por cada mil habitantes); la velocidad de cambio de éstas (si es que se dispone de datos para varios momentos en el tiempo; por ejemplo, la tasa de crecimiento de cierta patología); y las diferencias entre las áreas que integran el mapa (por ejemplo, índices de especialización local).

No obstante, los mapas temáticos tienen algunos problemas. Uno de ellos es que a menudo se representan las áreas como si sus 
límites fueran impenetrables, como si no hubiera libre tránsito de población entre ellas (casi como si fueran Estados nacionales). Cuando se trata de mapas de escala internacional que involucran diversos países, este supuesto puede ser correcto, pero no lo es cuando se trata de mapas de escala regional o intraurbana. En estos casos los mapas pueden presentar una imagen sesgada, sobre todo si se analiza la distribución de los recursos para la salud.

Por ejemplo, si suponemos una región (digamos, un estado de la República) en la que los servicios de salud se concentran en una sola unidad espacial (un solo municipio), el mapa correspondiente mostrará un municipio con una alta dotación de servicios, y al resto de los municipios con una disponibilidad igual a cero, lo que de ninguna manera refleja la situación real. En los hechos, los habitantes de los demás municipios acuden al que concentra los servicios para recibir atención. Por lo tanto, los habitantes del resto del estado sí disponen de servicios, aunque se localicen en municipios sin servicio. Sin embargo, la disponibilidad de recursos para la salud no es homogénea para todos los habitantes del estado, sino que varía inversamente con los costos de transporte (medidos en unidades económicas de tiempo, distancia o de incomodidades) que los separa de los servicios de salud. En otras palabras, la posibilidad de acceder a los recursos será mayor para los habitantes que enfrentan costos de transporte más bajos a los servicios de salud, que para los que enfrentan costos más altos. Esto explica por qué -cuando se analiza la distribución de los recursos de salud- se prefiere utilizar indicadores que consideren la "permeabilidad" de los límites de las diferentes unidades espaciales (Garrocho, 1993b), que los indicadores que simplemente registran la existencia de servicios en un cierto lugar. Los SIG facilitan el cálculo y la utilización operativa de indicadores espaciales, cuya expresión cartográfica resulta de gran utilidad como insumo de políticas de salud pública.

Otro problema de los mapas temáticos es que representan cada una de las áreas como si fueran homogéneas, cuando en realidad se registran importantes diferencias en su interior. ${ }^{5}$ Por lo tanto, hay que ser cautelosos en la interpretación de los mapas y recordar que son representaciones de la realidad, no la realidad misma.

Otros mapas muy populares son los de símbolos ${ }^{6}$ o cartogramas. A menudo se utilizan para mapear la localización y el

${ }^{5}$ Lo que los geógrafos llaman la "falacia ecológica".

' Por ejemplo, círculos proporcionales o cuadrados, triángulos, estrellas, y muchos más. tamaño de localidades o de grupos de población específica, o de eventos (por ejemplo, incidencias de cierta enfermedad o accidentes carreteros). Pero también para generar imágenes de la distribución e importancia de las unidades de salud. Estos mapas resultan muy útiles cuando se les asigna información a los símbolos. Por ejemplo, si se trata de localidades o de grupos de población, se pueden registrar datos de carácter demográfico, social, económico, de morbilidad, mortalidad, y otros que permitan tener un panorama más completo de las características de cada localidad o grupo, lo que sería útil para el diseño y monitoreo de políticas de salud pública.?

Cuando los símbolos representan unidades de salud se puede registrar información referente al equipo, personal, stock de medicamentos disponibles y diferentes indicadores de desempeño, lo que permite evaluar la calidad y actuación de las unidades existentes. Si además se combina el SIG con una base de datos ad hoc es posible incluir fotografías de las unidades de salud, lo que facilita tomar decisiones en materia de inversiones para el mantenimiento físico de las unidades. ${ }^{8}$

Además -como se asentó en la primera sección- los SIG pueden generar mapas a partir de otros mapas. Es decir, pueden empalmar y combinar varios mapas y generar nueva información cartográfica. Esta capacidad de los SIG es particularmente útil en los estudios de ecología médica y de epidemiología del paisaje. La superposición de mapas de factores de riesgo a la salud o de condiciones que favorecen el desarrollo de ciertos ciclos de enfermedad, permite identificar zonas de riesgo, lo que es sumamente útil para prevenir enfermedades de carácter vectorial.

ii. ¿Cuál es la mejor manera de distribuir socioespacialmente los recursos para la salud?

Dado que los recursos para la salud son importantes para el bienestar de la población, es pertinente preguntarse cuál es la mejor manera de

' Equivaldría a los "análisis de demanda" que se incluyen en los estudios de mercado del sector privado.

${ }^{8}$ En el Laboratorio de Análisis Socioespacial (LANSE) de El Colegio Mexiquense se han combinado mapas, bases de datos y fotografías de unidades escolares en un mismo SIC (Garrocho y Álvarez, 1995). 
distribuirlos, tanto en términos sociales como espaciales. ${ }^{9}$ Definir la distribución socioespacial de los recursos no es fácil, porque implica, primero, profundos análisis y discusiones morales relacionadas con la justicia distributiva $y$, segundo, diseñar y construir elaborados modelos matemáticos para hacer operativos los acuerdos morales (Garrocho, 1995b; 1997b).

Usualmente los SIG no incluyen modelos matemáticos de localización, pero sí algunas utilerías que permiten hacer planteamientos iniciales de política locacional. Por ejemplo, los SIG pueden trazar círculos de diámetro variable alrededor de puntos específicos en un mapa. Si se conoce el área de influencia normativa (o área de mercado) de las unidades de salud, y se le indica al SIG la localización de la demanda y la de las unidades de salud existentes en una región, se pueden trazar círculos alrededor de cada unidad, lo que simula sus áreas de influencia. Es decir, se puede asumir -en un primer momento- que la población localizada en las áreas definidas por los círculos está cubierta por el servicio, mientras que el resto de la población no, ya que acudir a las unidades de salud les exigiría costos demasiado elevados o socialmente injustos e inaceptables (en términos de tiempo, energía, incomodidades y dinero). Por lo tanto, las nuevas unidades de salud deberían localizarse en aquellas localidades no cubiertas por el servicio. Esta misma metodología puede seguirse para identificar fallas de integración entre unidades de salud de diferentes niveles jerárquicos, $\mathrm{y}$ así ayudar a reducir problemas de referencia y contrarreferencia de pacientes.

Por otro lado, los SIG podrían ser útiles también en la asignación de la población a cada unidad de salud. Por ejemplo, a partir de las utilerías gráficas de los SIG es relativamente sencillo definir en un mapa las unidades de servicios de salud más cercanas a cada localidad en una región dada, para luego definir la segmentación del mercado que más convenga en términos sociales.

Si de cualquier manera se requiere un método más refinado de localización de unidades de salud y de asignación de la población a las unidades, se podría programar un modelo matemático de localizaciónasignación e implantarlo como utilería del software. La experiencia indica que esto reporta ventajas, ya que las capacidades de cálculo de

' Dado que lo social y lo espacial están íntimamente ligados, lo más correcto sería hablar de la distribución socioespacial de los recursos para la salud: desde consultorios rurales (Garrocho, 1990a, 1990b), hasta clínicas, servicios de emergencia y hospitales especializados (Garrocho, 1995b) los SIG facilitan notablemente la utilización operativa de los modelos (Garrocho y Álvarez, 1995). ${ }^{10}$

Los modelos de asignación tradicionales tienen algunos problemas conceptuales, ya que asignan la totalidad de la población de las localidades analizadas a las unidades de servicio más cercanas. Esta regla de asignación (llamada de "todo o nada") no refleja el comportamiento espacial de los usuarios. En la realidad, los pacientes pueden acudir a diversas unidades de salud y no necesariamente a la más cercana (Phillips, 1979; Garrocho, 1992). Por lo tanto, los modelos de asignación recientes se fundamentan en la teoría de la interacción espacial.

Los modelos de interacción espacial permiten simular flujos de pacientes entre áreas y unidades de salud, o entre unidades de salud de diferente nivel jerárquico. Esto es importante en estudios prospectivos o de impacto, para anticipar los flujos de pacientes a unidades de salud específicas y prever problemas de sobre o subutilización de los servicios (Garrocho, 1995a). No es raro que los SIG incluyan modelos de interacción espacial muy elementales (llamados "gravitacionales"), pero que resultan poco recomendables para estudios rigurosos. Por lo tanto, la mayoría de las veces es necesario programarlos especialmente e instrumentarlos en el ambiente del SIG que se esté utilizando. ${ }^{11}$

Por otro lado, si se trata de hacer geografía médica prospectiva, resultará fundamental anticipar la distribución espacial de la población (la demanda). Los SIG no incluyen modelos de simulación de crecimiento urbano, pero una revisión de mapas con información demográfica para diferentes momentos permitiría anticipar los nuevos patrones de poblamiento. Por ejemplo, al examinar cartográficamente la distribución de población de la zona metropolitana de la ciudad de México (ZMCM) entre 1950 y 1970 , resulta evidente que la demanda por servicios de salud en su parte central ha disminuido sistemáticamente, y lo más probable es que la tendencia se mantenga durante los próximos años; en cambio, la población se incrementará aceleradamente en algunos municipios del

${ }^{10}$ En el Laboratorio de Análisis Socioespacial de El Colegio Mexiquense se han desarrollado varios modelos de localización-asignación en ambiente SIG y las pruebas han sido muy alentadoras.

"En el Laboratorio de Análisis Socioespacial de El Colegio Mexiquense se ha desarrollado un paquete de más de diez modelos de interacción espacial, que funciona como utilería de MAPINFO. El paquete no sólo permite calcular flujos probables entre unidades espaciales, sin que puede representar gráficamente su dirección y magnitud en una base cartográfica. 
Estado de México (Garrocho, 1996). Una política prospectiva de salud pública para la ZMCM debería tomar en cuenta estos cambios locacionales de la demanda potencial.

iii. ¿Cómo se explica el comportamiento espacial de los usuarios de servicios de salud?

Esta pregunta se relaciona con determinar los costos de transporte que la población está dispuesta a enfrentar para acceder a servicios de salud específicos, y -a un nivel de mayor complejidad-con identificar las razones que tienen los pacientes para acudir a ciertos 'servicios de salud y no a otros. Analizar el comportamiento espacial de los pacientes es fundamental para entender los patrones de utilización de los servicios de salud.

Esta pregunta de la geografía médica involucra numerosos factores, y sólo algunos son de naturaleza espacial. Por lo tanto, los SIG sólo podrían ofrecer un apoyo marginal. Por ejemplo, para identificar las áreas de mercado de las unidades de salud y calcular la sensibilidad de la demanda sobre cierto tipo de servicios ante cambios en los costos de transporte que se tienen que enfrentar para acceder a ellos. ${ }^{12}$

Así, a partir de los datos de utilización de cierto servicio (los registros médicos son una buena fuente de información al respecto) se puede construir un mapa que registre los lugares de residencia de los pacientes. Usualmente se define como área de mercado aquella que cubre a $80 \%$ de los pacientes registrados en los datos de utilización (Ghosh y McLafferty, 1987). Esto es algo que se puede hacer fácilmente con un SIG, y - como ya se dijo- es un dato básico para definir zonas no cubiertas por los servicios de salud, así como para dentificar las zonas y grupos de demanda real, es decir, los grupos de población que efectivamente utilizan los servicios. A partir de esta información se pueden formular algunas preguntas -y eventualmente algunas políticas-importantes en materia de salud pública: ¿por qué grupos similares localizados en la misma región utilizan los servicios con diferente intensidad?; ¿ sse detecta sobreutilización o subutilización de los servicios en zonas específicas?; ¿es posible tomar medidas -en

\footnotetext{
${ }^{12}$ Lo que en los análisis de interacción espacial se llama "fricción de la distancia".
}

lugares concretos- para evitar la sobre o subutilización de los servicios existentes?. ${ }^{13}$

Si se define el área de mercado de cierto servicio, es posible - con la ayuda de un SIG- contabilizar los usuarios a diferentes distancias de la unidad de salud, lo que serviría para calcular la fricción de la distancia de ese tipo de servicio. Este dato es fundamental en las simulaciones generadas a partir de modelos de interacción espacial.

Otra importante aplicación de los SIG para entender los patrones de utilización de los servicios de salud es mapear la distribución temporal de las unidades. Por ejemplo, al mapear los servicios públicos de salud disponibles por las tardes, noches y fines de semana en el Estado de México, resultó evidente la razón de la sobreutilización de los servicios de salud del hospital del DIF en la ciudad de Toluca (Garrocho, 1995a).

No obstante, la gran mayoría de las variables que explican los patrones de utilización de los servicios de salud no son de carácter espacial, por lo que vale recalcar que la utilidad de los SIG en este campo es limitada.

iv. ¿Cómo se explica la difusión espacial de las enfermedades y de las innovaciones en medicina?

Las enfermedades y las innovaciones en medicina se difunden en el espacio y en el tiempo. Diversos geógrafos médicos se han interesado por explicar tanto los procesos de difusión de agentes infecciosos, como la difusión de nuevas tecnologías, técnicas de diagnóstico o tratamientos médicos.

Existen diversos modelos -tanto discursivos como matemáticos- para explicar la difusión espacial y temporal de fenómenos sociales. Pero no son comúnmente incluidos como utilerías en los SIG. Se podría intentar simular en SIG procesos de difusión que siguieran la lógica de la teoría de interacción espacial, pero no es algo que se haga frecuentemente. De cualquier manera, no sería particularmente complicado adaptar modelos cuantitativos de difusión a un ambiente SIG.

${ }^{3}$ Si la idea fuese rebasar el nivel meramente descriptivo y avanzar algunas explicaciones de los diferentes patrones de utilización, tal vez sería necesario aplicar algunas técnicas y modelos de estadística espacial para calcular correlaciones, residuales, o componentes principales. 
Es más común, en cambio, empalmar diferentes mapas que registren momentos distintos de procesos de difusión y tratar de derivar conclusiones a partir de su inspección visual, o con base en cálculos de estadística espacial (pero tampoco son comunes las rutinas de este tipo en los SIG). En el primer caso, sería como comparar diferentes fotos (situaciones estáticas) para interpolar las imágenes intermedias e imaginar la secuencia completa del proceso de difusión. En el segundo, se trataría de utilizar modelos o herramientas de estadística espacial que permitieran sintetizar la información de uno o varios mapas, y darle sentido a los datos.

v. ¿Por qué los individuos perciben de manera diferente su salud y las medidas que pueden tomar para mantenerla o mejorarla?

La percepción individual y colectiva de las causas y gravedad de las enfermedades definen las acciones que se tomen para restablecer la salud. Si además existen varios tipos de servicios de salud (privados, públicos, tradicionales, modernos), las percepciones definirán qué servicio de salud es el más adecuado en cada situación y qué acciones se pueden y deben realizar para restablecerla.

En este tipo de investigaciones se incluyen numerosas variables de carácter social y cultural, y pocas de corte espacial, y por lo tanto la utilidad de los SIG es mínima. Vale la pena subrayar cómo la geografía médica ha sabido rebasar el rígido armazón de lo espacial para incorporar lo social en el más amplio sentido del término: involucrando en sus análisis numerosas variables sociales, culturales, económicas, políticas, antropológicas, psicológicas, y otras. Esta pregunta de investigación -las demás también, pero ésta particularmente - ilustra la complejidad y potencialidad analítica de la geografía médica moderna.

\section{Conclusiones}

La geografía médica es una las disciplinas más complejas y apasionantes en las ciencias sociales. Al intentar integrar las relaciones sociales y las estructuras espaciales, ofrece una plataforma analítica sólida para explicar diversos aspectos del proceso salud-enfermedad. La plataforma socioespacial de la moderna geografía médica enriquece la disciplina y define el papel de los SIG como herramienta de apoyo en la investigación geográfica de la salud, la enfermedad, y los recursos para la salud: la utilidad de los SIG para apoyar la investigación en geografía médica, es proporcional a la importancia del espacio en las preguntas de investigación.

Es decir, mientras más importante sea la dimensión espacial de las preguntas de investigación, más relevante será el uso de SIG pero a mayor importancia de la dimensión social, menos pertinente será usarlos. Así de sencillo, pero así de complejo.

Es fácil deducir que la disciplina - como método de generar conocimiento- es mucho más poderosa en términos análiticos que una simple herramienta, por compleja que sea. Sería lamentable confundirlas y caer en el error de reducir la geografía médica a la simple utilización de un SIG. Como intenté ilustrarlo, la geografía médica contemporánea es mucho más que realizar mapas (por valiosos que sean); es más que identificar patrones (por interesantes que parezcan); es entender procesos y sugerir políticas (por pretencioso que resulte). Esto es el reto de la geografía en general, y de la geografía médica en particular.

Los SIG tienen un papel que jugar en esta empresa, pero siempre como herramienta subsidiaria de preguntas de investigación socialmente relevantes. Sólo así podrán ser útiles para apoyar la generación de conocimiento que nos ayude a vivir mejor y nos libere de la ignorancia.

\section{Bibliografía}

Álvarez, A. (1997), Diseño y construcción de un modelo operativo urbano: instrumentación del modelo de Garin-Lowry para el caso mexicano, México, Universidad Iberoamericana, tesis de maestría en sistemas, planeación e informática (mimeo).

Amedeo, D. y R. G. Golledge (1975), An Introduction to Scientific Reasoning in Geography, Nueva York, Wiley.

Bailey, T. (1994), "A review of statistical spatial analysis in geographical information systems", en S. Fotheringham y P. Rogerson, Spatial Analysis and GIS, Londres, Taylor y Francis.

Clarke, M. y A. Wilson (1989), "Mathematical models in human geography: 20 years on", en R. Peet y N. Thrift, New Models in Geography, Londres, Unwin Hyman. 
Frank, A.V. (1988), "Requirements for a data base management system for a GIS", Photogrammetric Engineering and Remote Sensing, núm. 54, pp. 1557-1564.

Garrocho, C. (1990a), "Servicios de salud y planeación regional en el Estado de México", Estudios Territoriales (España), vol. 33, pp. 55-1072.

(1990b), "Localización geográfica de los servicios de salud en un subsistema de asentamientos rurales: un intento de evaluación", Estudios Demográficos y Urbanos, El Colegio de México, vol. 5, núm. 1, pp. 127-148.

- (1992), Localización de servicios en la planeación urbana $y$ regional, Toluca, El Colegio Mexiquense.

- (1993a), "De la casa al hospital: un enfoque espacio-temporal", Estudios Sociológicos, El Colegio de México, vol. XI, núm. 32, mayo-agosto, pp. 547-554.

- (1993b), "Análisis de accesibilidad a los servicios de salud y los sistemas de información geográfica: teoría y aplicación en el contexto del Estado de México", Estudios Demográficos y Urbanos, El Colegio de México, vol. 8, núm. 2, pp. 427-444.

_ (1993c), "Eficiencia, igualdad y equidad en la localización de los servicios de salud infantil del Estado de México", Estudios Demográficos y Urbanos, El Colegio de México, vol. 8, núm. 3, pp. 601-640.

- (1995a), "Justicia social y planeación espacial de los servicios públicos", en C. Massé y E. Sandoval (coords.), Políticas públicas y desarrollo municipal, Toluca, El Colegio Mexiquense-UAEM.

(1995b), Análisis socioespacial de los servicios de salud: accesibilidad, utilización y calidad, Toluca, El Colegio Mexiquense.

(1996), "Distribución espacial de la población de la zona metropolitana de la Ciudad de México, 1950-1990", Estudios
Demográficos y Urbanos, El Colegio de México, vol. 11, núm. 31, pp. 69-100

(1997a), "SEIGO: una innovación técnica en el proceso de planeación de un gobierno local", en C. Garrocho y J. Sobrino (coords.), Desarrollo municipal: retos y posibilidades, Toluca, El Colegio Mexiquense (en prensa).

(1997b), "La articulación de lo moral y lo técnico en la planeación espacial de los servicios públicos de salud", Ciudades, núm. 33, pp. 59-63.

- y A. Álvarez (1995), "Efectos del nuevo aeropuerto internacional en la estructura metropolitana de Toluca", Comercio Exterior, vol. 45, núm. 10, pp. 786-796.

Ghosh, A. y S. McLafferty (1987), Location Strategies for Retail and Service Firms, Nueva York, Lexington Books.

Giggs, J. (1983), "Schizophrenia and ecological structure in Nottingham", en N. D. McGlashan y J. Blunden (eds.), Geographical Aspects of Health, Londres, Academic Press.

Huxhold, W.E., (1991), An Introduction to Geographic Information Systems, Oxford, Oxford University Press.

Joseph, A. y D. R. Phillips (1985), Accessibility and Utilization: Geographical Perspectives on Health Care Delivery, Londres, Harper and Row.

Learmonth, A. T. A. (1978), Patterns of Desease and Hunger, Newtton Abbot, David and Charles.

Maguire, D. J., M. F. Goodchild y D. W. Rhind (1991), Geographical Information Systems, Londres, Longman Scientific and Technical.

May, J. M. (1958), The Ecology of Human Desease, Nueva York, MD Publications.

McGlashan, N. D. (1966), "The medical geographer's work", International Patbology, núm. 7, pp. 81-83. 
Especificaciones para la entrega de los artículos de la revista Econo-

Meade, M., J. Florin y W. Gesler (1988), Medical Geography, Nueva York, The Guilford Press.

O'Kelly, M. E. (1994), "Spatial analysis and GIS", en S. Fotheringham y P. Rogerson, Spatial Analysis and GIS, Londres, Taylor y Francis.

Pavlovski, E. N. (1966), The Natural Nidality of TransmissibleDesease, Urbana il, University of Illinois Press.

Phillips, D. R. (1979), "Spatial variations in attendance at general practitioners services", Social Science and Medicine, núm. 13, pp. 169-181.

- (1981), Contemporary Issues in the Geography of Health Care, Norwich, Geo Books.

Pyle, G. F. (1976), "Foundations to medical geography", Economic Geography, núm. 52, pp. 95-102.

(1977), "International communication and medical geography”, Social Science and Medicine, núm. 11, pp. 679-682.

(1986), The Diffusion of Influenza, Totowa, Nueva Jersey, Rowman and Littlefield.

mia, Sociedad y Territorio.

La entrega de un trabajo para su publicación en la revista Economía, Sociedad y Territorio implica para el colaborador el compromiso de no someterlo a otras publicaciones en español al momento de su aprobación para su aparición dentro de la revista. La publicación admite textos en otros idiomas encargandose ésta de la traducción al español. En los casos en los cuales sean enviados en español se anexará un ejemplar en el idioma original.

Se sugiere que los artículos tengan una extensión de 15 cuartillas como mínimo y 40 como máximo, incluidas gráficas, cuadros, notas y bibliografía; las reseñas podrán tener entre 5 y 10 cuartillas; cada número de la revista esta compuesto de 280 cuartillas como máximo de extensión (incluyendo cuadros, gráficas y todo material gráfico que se incluya en el número).

La presentación de los textos deberá enmarcarse dentro de los siguientes lineamientos:

1.-Los artículos deberán de presentarse en su versión final y completas ya que no se admitiran cambios iniciado el proceso de producción de la publicación.

2.-Los textos se presentarán impresos en matriz de puntos o en laser a doble espacio $(1,5)$ en tipo curier o times de 11 puntos; los textos que sean entregados mecanografiados deberán estar a doble espacio en cuartillas de 28 líneas y aproximadamente 64 golpes por línea sobre papel grueso; por ningún motivo serán recibidos sobre papel copia y/ o copias al carbón. A estos-materiales se le sumará el artículo en procesador de palabras, de preferencia en un disquette de 3.5 y en Word perfect 5.1, Word 6 o almacenarse bajo código de TEXTO DOS (ASCII).

3.-Las referencias bibliográficas se harán en el texto, entre paréntesis, de acuerdo al siguiente modelo: apellido del autor(a), año de edición, número de la página.

Las notas a pie de página, los cuadros y las gráficas se agruparán al final del artículos después de la bibliografía. Las gráficas y cuadros deberán presentarse impresos en originales claros y precisos que 
permitan su publicación; en el caso de ser enviados en disquette deberán de estar almacenados en Excel 5 de ninguna manera seránrecibidos en otro programa o versiones posteriores a ésta.

En el texto deberá de indicarse la colocación aproximadamente de estos materiales gráficos.

La lista de referencias bibliográficas deberan presentarse completas y en el siguiente orden: apellido y nombre del autor(a) año de edición, nombre del libro, país o ciudad, editorial.

ejemplo:

Legorreta, Jorge (1992), "Expansión urbana de la ciudad de México" en A. Bassols, J. Delgadillo y F. Torres (comps.), El desarrollo regional en México: Teoría y práctica, México, IIE, UNAM:

4.-La página inicial de cada artículo deberá contener: a) resumen en español con extensión máxima de 200 palabras.; b) nombre del autor(a), su filiación institucional, grados académicos, área de interés principal y dos referencias bibliográficas de su autoría; c) número telefónico (fax), dirección electrónica (E-mail) y dirección postal para su eventual localización.

5.-Los artículos y su manejo son competencia del coordinador responsable del número al cual los autores tendrán que dirigirse para cualquier duda o aclaración.

\section{El Colegio Mexiquense, A.C.}
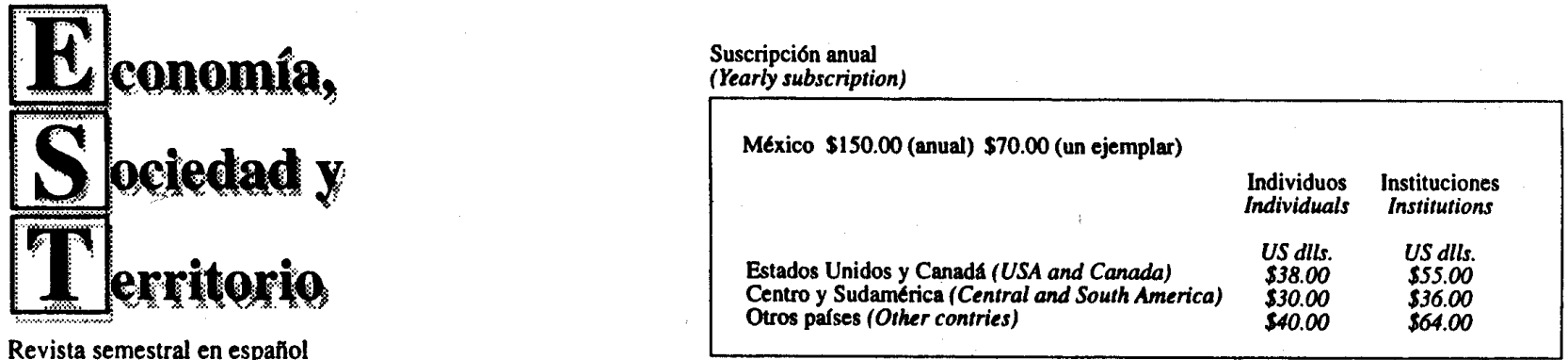

Revista semestral en español

(A journal published twiece a year in Spanish)

Adjunto cheque o giro bancario a nombre de El Colegio Mexiquense, A.C., núm. (Enclosed bank check or order payable to)

Banco
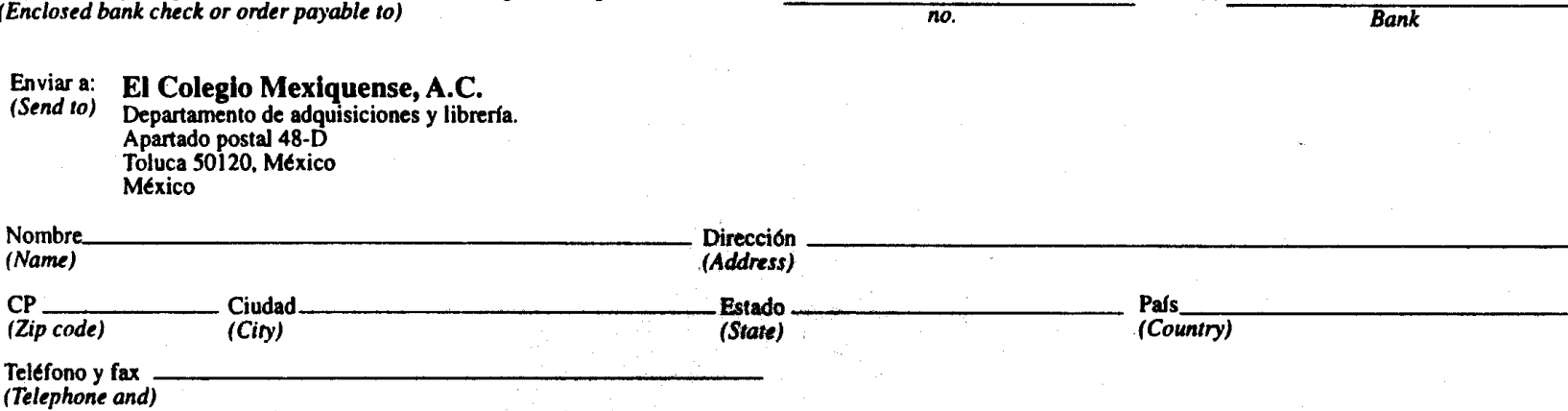